ISSN 2075-9827 e-ISSN 2313-0210

Carpathian Math. Publ. 2018, 10 (2), 288-295

doi:10.15330/cmp.10.2.288-295 http://www.journals.pu.if.ua/index.php/cmp

Карпатські матем. публ. 2018, Т.10, №2, С.288-295

DEhGHANi M.A., DaVvaz B.

\title{
ON CENTRAL AUTOMORPHISMS OF CROSSED MODULES
}

\begin{abstract}
A crossed module $(T, G, \partial)$ consist of a group homomorphism $\partial: T \rightarrow G$ together with an action $(g, t) \rightarrow{ }^{g} t$ of $G$ on $T$ satisfying $\partial\left({ }^{g} t\right)=g \partial(t) g^{-1}$ and ${ }^{\partial(s)} t=s t s^{-1}$, for all $g \in G$ and $s, t \in T$. The term crossed module was introduced by J. H. C. Whitehead in his work on combinatorial homotopy theory. Crossed modules and its applications play very important roles in category theory, homotopy theory, homology and cohomology of groups, algebra, K-theory etc. In this paper, we define Adeny-Yen crossed module map and central automorphisms of crossed modules. If $C^{*}$ is the set of all central automorphisms of crossed module $(T, G, \partial)$ fixing $Z(T, G, \partial)$ element-wise, then we give a necessary and sufficient condition such that $C^{*}=I_{n n}(T, G, \partial)$. In this case, we prove $\operatorname{Aut}_{C}(T, G, \partial) \cong \operatorname{Hom}((T, G, \partial), Z(T, G, \partial))$. Moreover, when $\left.\operatorname{Aut}_{C}(T, G, \partial) \cong Z\left(I_{n n}(T, G, \partial)\right)\right)$, we obtain some results in this respect.

Key words and phrases: crossed module, central automorphism.
\end{abstract}

Department of Mathematics, Yazd University, P.O. Box 89195-741, Yazd, Iran

E-mail: dehghani19@yahoo.com (Dehghani M.A.), davvaz@yazd.ac.ir (Davvaz B.)

\section{INTRODUCTION}

The term crossed module was introduced by J. H. C. Whitehead in his work on combinatorial homotopy theory [9]. So many mathematicians and many areas of mathematics have used crossed modules such as homotopy theory, homology and cohomology of groups, algebra, K-theory etc. Actor crossed module of algebroid was defined by Alp in [3]. Actions and automorphisms of crossed modules were studied by K. Norrie [2,8]. Tensor product modulo $q$ of two crossed modules defined by Conduché and Rodriguez-Fernandez [4]. Concepts of $q$-commutator and of $q$-center of a crossed module, $q$ being a nonnegative integer, were defined by J.L. Doncel Juurez and A.R. Crondjeanl.-Valcarcel [6]. Adney and Yen in [1] obtained several sufficient conditions for a non-abelian $p$-group and introduced a special map. By using Adney-Yen map, in this paper, we introduce the concept of Adney-Yen crossed module map and central automorphisms of a crossed module and obtain some results in this respect.

\section{Central AUtOMORPHisms of A CROSSED MOdUle}

We recall some basic definitions and properties of the category of crossed modules. A crossed module $(T, G, \partial)$ consists of a group homomorphism $\partial: T \rightarrow G$ called the boundary map, together with an action $(g, t) \rightarrow g_{t}$ of $G$ on $T$ satisfying (1) $\partial\left(g_{t} t\right)=g \partial(t) g^{-1}$ and (2) $\partial(s) t=s t s^{-1}$, for all $g \in G$ and $s, t \in T$. 
The group automorphism Aut $N$ of a group $N$ comes equipped with the canonical homomorphism $\tau: N \rightarrow \operatorname{Aut}(N)$ which has image $\operatorname{Inn} N$, the group of inner automorphism of $N$. The inner automorphism $\tau$ is one of the standard examples of a crossed module. Other standard examples of crossed modules are: the inclusion of a normal subgroup $N \rightarrow G$; a $G$-module $M$ with the zero homomorphism $M \rightarrow G$; any epimorphism $E \rightarrow G$ with central kernel. We note at once certain consequences of the definition of a crossed module: (1) the kernel kerd lies in $Z(T)$, the center of $T$; (2) the image $\partial(T)$ is a normal subgroup of $G$; (3) the action of $G$ on $T$ induces a natural $(G / \partial(T))$-module structure on $Z(T)$; and kerd is a submodule of $Z(T)$.

We say that $\left(S, H, \partial^{\prime}\right)$ is a sub-crossed module of the crossed module $(T, G, \partial)$ if

- $S$ is a subgroup of $T$, and $H$ is a subgroup of $G$;

- $\partial^{\prime}$ is the restriction of $\partial$ to $S$;

- the action of $H$ on $S$ is included by the action of $G$ on $T$.

A sub-crossed module $(S, H, \partial)$ of $(T, G, \partial)$ is normal if

- $H$ is a normal subgroup of $G$;

- $g_{S} \in S$ for all $g \in G, s \in S$;

- ${ }^{h} t t^{-1} \in S$ for all $h \in H, t \in T$.

In this case we consider the triple $(T / S, G / H, \bar{\partial})$, where $\bar{\partial}: T / S \rightarrow G / H$ is induced by $\partial$, and the new action is given by $g H(t S)=(g t) S$. This is the quotient crossed module of $(T, G, \partial)$ by $(S, H, \partial)$. A crossed module morphism $\langle\alpha, \varphi\rangle:(T, G, \partial) \rightarrow\left(T^{\prime}, G^{\prime}, \partial^{\prime}\right)$ is a commutative diagram of homomorphisms of groups

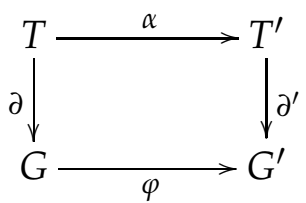

such that for all $x \in G$ and $t \in T$; we have $\alpha\left({ }^{x} t\right)=\varphi(x) \alpha(t)$. We say that $\langle\alpha, \varphi\rangle$ is an isomorphism if $\alpha$ and $\varphi$ are both isomorphisms. We denote the group of automorphisms of $(T, G, \partial)$ by $\operatorname{Aut}(T, G, \partial)$. The kernel of the crossed module morphism $\langle\alpha, \varphi\rangle$ is the normal sub-crossed module $(\operatorname{ker} \alpha, \operatorname{ker} \varphi, \partial)$ of $(T, G, \partial)$, denoted by $\operatorname{ker}\langle\alpha, \varphi\rangle$. The image $\operatorname{im}\left\langle\mathrm{ff},{ }^{\prime}\right\rangle$ of $\langle\alpha, \varphi\rangle$ is the sub-crossed module (imff, im', $\left.\partial^{\prime}\right)$ of $\left(T^{\prime}, G^{\prime}, \partial^{\prime}\right)$. For a crossed module $(T, G, \partial)$, denote by $\operatorname{Der}(G, T)$ the set of all derivations from $G$ to $T$, i.e., all maps $\chi: G \rightarrow T$ such that for all $x, y \in G, \chi(x y)=\chi(x)^{x} \chi(y)$. Each such derivation $\chi$ defines endomorphisms $\sigma=\left(\sigma_{x}\right)$ and $\theta\left(=\theta_{x}\right)$ of $G, T$ respectively, given by $\left.\sigma(x)\right)=\partial \chi(x) x$ and $\theta(t)=\chi \partial(t) t$, where $\sigma \partial(t)=\partial \theta(t)$, $\theta \chi(x)=\chi \partial(x)$ and $\theta\left({ }^{x} t\right)={ }^{\sigma(x)} \theta(t)$. We define a multiplication in $\operatorname{Der}(G, T)$ by the formula $\chi_{1} \circ \chi_{2}=\chi$, where $\chi(x)=\chi_{1} \sigma_{2}(x) \chi_{2}(x)\left(=\theta_{1} \chi_{2}(x) \chi_{1}(x)\right)$. This turns $\operatorname{Der}(G, T)$ into a semigroup with identity element the derivation which maps each element of $G$ into identity element of $T$. Moreover, if $\chi=\chi_{1} \circ \chi_{2}$, then $\sigma=\sigma_{1} \sigma_{2}$. The whitehead group $D(G, T)$ is defined to be the group of units of $\operatorname{Der}(G, T)$, and the elements of $D(G, T)$ are called regular derivations. 
Proposition 1. The following statements are equivalent: (1) $\chi \in D(G, T)$; (2) $\sigma \in \operatorname{Aut}(G)$; (3) $\theta \in \operatorname{Aut}(T)$.

The map $\Delta: D(G, T) \rightarrow \operatorname{Aut}(T, G, \partial)$ defined by $\Delta(X)=\langle\sigma, \theta\rangle$ is a homomorphisms of groups and there is an action of $\operatorname{Aut}(T, G, \partial)$ on $D(G, T)$ given by ${ }^{\langle\alpha, \varphi\rangle} \chi=\alpha \chi \varphi^{-1}$ which makes $(D(G, T), \operatorname{Aut}(T, G, \partial), \Delta)$ a crossed module. This crossed module is called the actor crossed module $\mathcal{A}(T, G, \partial)$ of the crossed module $(T, G, \partial)$. There is a morphism of crossed modules $\langle\eta, \gamma\rangle:(T, G, \partial) \rightarrow \mathcal{A}(T, G, \partial)$ defined as follows. If $t \in T$, then $\eta_{t}: G \rightarrow T$ defined by $\eta_{t}(x)=t^{x} t^{-1}$ is a derivation and the map $t \rightarrow \eta_{t}$ defines a homomorphism $\eta: T \rightarrow D(G, T)$ of groups. Let $\gamma: G \rightarrow \mathcal{A}(T, G, \partial)$ be the homomorphism $y \rightarrow\left\langle\alpha_{y}, \varphi_{y}\right\rangle$, where $\alpha_{y}(t)=y_{t}$ and $\varphi_{y}(x)=y x y^{-1}$ for $t \in T$ and $y, x \in G$.

Definition 1. Let $(T, G, \partial)$ be a crossed module. The center of $(T, G, \partial)$ is the crossed module kernel $Z(T, G, \partial)$ of $\langle\eta, \gamma\rangle$. Thus, $Z(T, G, \partial)$ is the crossed module $\left(T^{G}, S t_{G}(T) \cap Z(G), \partial\right)$, where $T^{G}$ denotes the fixed point subgroup of $T$, that is, $T^{G}=\left\{t \in T \mid{ }^{x} t=t\right.$ for all $\left.x \in G\right\}$. $S t_{G}(T)$ is the stabilizer in $G$ of $T$, that is, $S t_{G}(T)=\left\{x \in G \mid{ }^{x} t=t\right.$ for all $\left.t \in T\right\}$ and $Z(T)$ is the center of $G$. Note that $T^{G}$ is central in $T$.

Definition 2. Let $(T, G, \partial)$ be a crossed module. $n$-center of $(T, G, \partial), Z^{n}(T, G)$ for $n$ a nonnegative integer is the crossed module $\left(\left(T^{G}\right)^{n}, Z^{n}(G) \cap S t_{G}(T), \partial\right)$, where

$$
\begin{aligned}
\left(T^{G}\right)^{n} & =\left\{t \in T \mid t^{n}=1 \text { and }{ }^{g} t=t \text { for all } g \in G\right\}, \\
Z^{n}(G) & =\left\{g \in Z(G) \mid g^{n}=1\right\}, \\
S t_{G}(T) & =\left\{\left.g \in G\right|^{g} t=t \text { for all } t \in T\right\} .
\end{aligned}
$$

The $n$-center of $(T, G, \partial)$ is a normal crossed submodule and is called the $n$-central crossed submodule of $(T, G, \partial)$.

Let $(T, G, \partial)$ be a crossed module, $\left(T^{\prime}, G^{\prime}, \partial\right)$ be a normal sub-crossed module of it, and $\langle\alpha, \varphi\rangle \in \operatorname{Aut}(T, G, \partial)$. Then $\langle\alpha, \varphi\rangle$ induces a $\langle\bar{\alpha}, \bar{\varphi}\rangle$ in Aut $\left(T / T^{\prime}, G / G^{\prime}, \bar{\partial}\right)$ such that $\bar{\partial}: T / T^{\prime} \rightarrow$ $G / G^{\prime}, \bar{\partial}\left(t T^{\prime}\right)=\partial(t) T^{\prime}$.

Definition 3. Let $(T, G, \partial)$ be a crossed module, $Z(T, G)$ be the center of it and $\langle\alpha, \varphi\rangle \in$ $\operatorname{Aut}(T, G, \partial)$. If $\langle\bar{\alpha}, \bar{\varphi}\rangle$, induced by $\langle\alpha, \varphi\rangle$ in $\operatorname{Aut}\left(T / T^{G}, G / S t_{G}(T) \cap Z(G), \bar{\partial}\right)$, is identity, then $\langle\alpha, \varphi\rangle$ is called central automorphism of crossed module $(T, G, \partial)$.

Theorem 1. If $(T, G, \partial)$ has trivial $n$-center, then its actor $\mathcal{A}(T, G, \partial)$ also has trivial $n$-center.

Proof. Let us assume that $Z^{n}(T, G, \partial)=1$ so that $\left(T^{G}\right)^{n}=1$ and $Z^{n}(G) \cap S t_{G}(T)=1$. Now the $n$-center of $\mathcal{A}(T, G, \partial)$ is the crossed module

$$
\begin{aligned}
\mathcal{A}(T, G, \partial) & =(D(G, T), \operatorname{Aut}(T, G, \partial), \Delta), Z^{n}(\mathcal{A}(T, G, \partial)) \\
& =\left(\left(D(G, T)^{\operatorname{Aut}(T, G, \partial)}\right)^{n}, Z^{n}(\operatorname{Aut}(T, G, \partial)) \cap S t_{\operatorname{Aut}(T, G, \partial)}(D(G, T), \Delta) .\right.
\end{aligned}
$$

So, assume that $\chi \in\left(D(G, T)^{\operatorname{Aut}(T, G, \partial)}\right)^{n}$. Then, for all $\langle\alpha, \varphi\rangle \in \operatorname{Aut}(T, G, \partial),\langle\alpha, \varphi\rangle \chi=\chi$ and $\chi^{n}=1$. In particular, this is true for all $\left\langle\alpha_{y}, \varphi_{y}\right\rangle$, where $y \in G$. But $\left\langle\alpha_{y}, \varphi_{y}\right\rangle_{\chi}=\eta_{\chi(y)^{-1}} \circ \chi$. So $\left\langle\alpha_{y}, \varphi_{y}\right\rangle \chi=\chi$ implies that $\eta_{\chi(y)^{-1}}=1$ for all $y \in G$. Then, we have $\chi(y)^{-1 x} \chi(y)=1$, for all 
$x, y \in G$. Now, since $\left(T^{G}\right)^{n}=1, \chi$ is the trivial derivation, it follows that $\left(D(G, T)^{\left\langle\alpha_{y}, \varphi_{y}\right\rangle}\right)^{n}=1$. Now, suppose that $\langle\alpha, \partial\rangle \in Z^{n}(\operatorname{Aut}(T, G, \partial)) \cap S t_{\operatorname{Aut}(T, G, \partial)}(D(G, T))$. Then ${ }^{\langle\alpha, \varphi\rangle} \chi=\chi$ for all $\chi D(G, T)$. In particular, $\langle\alpha, \varphi\rangle \eta_{t}=\eta_{t}$, for all $t \in T$, that is $\eta_{\alpha(t)}=\eta_{t}$, which implies that $t^{-1} \alpha(t) \in\left(T^{G}\right)^{n}=1$, for all $t \in T$. Thus $\alpha=1_{T}$, the identity automorphism of $T$. Now we have $\langle\alpha, \varphi\rangle \in Z^{n}(\operatorname{Aut}(T, G, \partial))$. Hence, for all $y \in G,\langle\alpha, \varphi\rangle\left\langle\alpha_{y}, \varphi_{y}\right\rangle=\left\langle\alpha_{y}, \varphi_{y}\right\rangle\langle\alpha, \varphi\rangle$ and $\langle\alpha, \varphi\rangle^{n}=1$ implying that $\varphi \varphi_{y}=\varphi_{y} \varphi$ for all $y \in G$. So we obtain $\varphi\left(x y x^{-1}\right)=y \varphi(x) y^{-1}$ for all $x, y \in G$. Since $\varphi$ is an automorphism of $G$, it follows that $y^{-1} \varphi(y) \in Z(G)$, for all $y \in G$. Now, since $\langle\alpha, \varphi\rangle$ is a crossed module morphism, it follows that $\alpha\left({ }^{y} t\right)=\varphi(y) \alpha(t)$. But $\alpha^{n}=1_{T}$ so that ${ }^{y} t=\varphi(y) t$ for all $y \in G$ and $t \in T$. Thus, $y^{-1} \varphi(y) \in Z^{n}(G) \cap S t_{G}(T)=1$ so that $\varphi^{n}=1_{G}$. Therefore $\left\langle\alpha^{n}, \varphi^{n}\right\rangle=\left\langle 1_{T}, 1_{G}\right\rangle$, and this completes the proof.

A non-abelian group that has no non-trivial abelian direct factor is said to be purely nonabelian [1].

By using Adney-Yen map [1], we introduce the following definition.

Definition 4. An Adney-Yen crossed module map is an onto map

$$
\left\langle\varphi_{1}, \varphi_{2}\right\rangle \text { from } \operatorname{Aut}_{C}(T, G, \partial) \text { to } \operatorname{Hom}((T, G, \partial), Z(T, G, \partial))
$$

such that $\left\langle\varphi_{1}, \varphi_{2}\right\rangle\langle\alpha, \theta\rangle=\left\langle\varphi_{1}, \varphi_{2}\right\rangle\langle\alpha, \theta\rangle$ and $\left\langle\varphi_{1}, \varphi_{2}\right\rangle\langle\alpha, \theta\rangle$ is the crossed module homomorphism of $(T, G, \partial)$ into $Z(T, G, \partial)=\left(T^{G}, S t_{G}(T) \cap Z(G), \partial\right)$. Furthermore, such that $\left\langle\varphi_{1}, \varphi_{2}\right\rangle\langle\alpha, \theta\rangle=$ $\left\langle\varphi_{1\langle\alpha, \theta\rangle}, \varphi_{2}\langle\alpha, \theta\rangle\right\rangle$, where $\varphi_{1\langle\alpha, \theta\rangle}: T \rightarrow T^{G}, \varphi_{1\langle\alpha, \theta\rangle}(t)=t^{-1} \alpha(t)$ and $\varphi_{2}\langle\alpha, \theta\rangle: G \rightarrow S t_{G}(T) \cap$ $Z(G), \varphi_{2\langle\alpha, \theta\rangle}(g)=g^{-1} \theta(g)$.

Theorem 2. For purely non-abelian groups $T$ and $G$ an Adeny-Yen crossed module map is one-to-one correspondence of $A u t_{C}(T, G, \partial)$ onto Hom $((T, G, \partial), Z(T, G, \partial))$.

Proof. The crossed module map $\langle\alpha, \theta\rangle \rightarrow\left\langle\varphi_{1}, \varphi_{1}\right\rangle_{\langle\alpha, \theta\rangle}$ is a one-to-one crossed module map of $\operatorname{Aut}_{C}(T, G, \partial)$ into the Hom $((T, G, \partial), Z(T, G, \partial))$.

Conversely, if $\left\langle f_{1}, f_{2}\right\rangle \in \operatorname{Hom}((T, G, \partial), Z(T, G, \partial))$, then $\left\langle\varphi_{1}, \varphi_{2}\right\rangle_{\left\langle f_{1}, f_{2}\right\rangle}(t)=t f_{1}(t)$, for all $t \in T$ and $\left\langle\varphi_{1}, \varphi_{2}\right\rangle\left\langle f_{1}, f_{2}\right\rangle(g)=g f_{2}(g)$, for all $g \in G$, defines an endomorphism of $(T, G, \partial)$. The endomorphism $\left\langle\varphi_{1}, \varphi_{2}\right\rangle_{\left\langle f_{1}, f_{2}\right\rangle}$ is an automorphism if and only if $f_{1}(t) \neq t^{-1}$ and $f_{2}(g) \neq$ $g^{-1}$ for every $g \in G, g \neq 1$ and $t \in T, t \neq 1$. But if $T$ and $G$ are direct product with an abelian factor, then there exists $\left\langle f_{1}, f_{2}\right\rangle \in \operatorname{Hom}((T, G, \partial), Z(T, G, \partial))$ such that $\left\langle f_{1}, f_{2}\right\rangle\langle t, g\rangle=$ $\langle t, g\rangle^{-1}$, for some $g \in G, g \neq 1$ and $t \in T, t \neq 1$. So, suppose that there exists $\left\langle f_{1}, f_{2}\right\rangle \in$ $\operatorname{Hom}((T, G, \partial), Z(T, G, \partial))$ such that $f_{1}(t)=t^{-1}$ for some $t \in T, t \neq 1$ and $f_{2}(g)=g^{-1}$ for some $g \in G, g \neq 1$. Clearly, $t \in Z(T), g \in Z(G)$. We assume that the order of $t, o(t)=p_{1}$ and $o(g)=p_{2}$ such that $p_{1}, p_{2}$ are primes. If

$$
T / T^{\prime}=T_{p_{1}} / T^{\prime} \times T_{p_{1}}^{\prime} / T^{\prime} \text { and } G / G^{\prime}=G_{p_{2}} / G^{\prime} \times G_{p_{2}}^{\prime} / G^{\prime},
$$

where $T_{p_{1}} / T^{\prime}$ is the $p_{1}$-primary component of $T / T^{\prime}$ and $G_{p_{2}} / G^{\prime}$ is the $P_{2}$-primary component of $G / G^{\prime}$, then $t T^{\prime} \in T_{p_{1}} / T^{\prime}, t T^{\prime} \neq T^{\prime}$ and $g G^{\prime} \in G_{p_{2}} / G^{\prime}, g G^{\prime} \neq G^{\prime}$ for is contained in the kernel of $f_{1}$ and $T^{\prime}$ is contained in the kernel of $f_{2}$. But if the height of $t T^{\prime}$ in $T_{p_{1}} / T^{\prime}$ be $p_{1}^{k_{1}}$ and $t=x_{1} p_{1}^{k_{1}} u$, where $x_{1} \in T_{p_{1}}$ and $u \in T^{\prime}, g G^{\prime}$ in $G_{p_{2}} / G^{\prime}$ be $p_{2}{ }^{k_{2}}$ and $g=x_{2} p_{2}^{k_{2}} v$, where $x_{2} \in G_{p_{2}}$

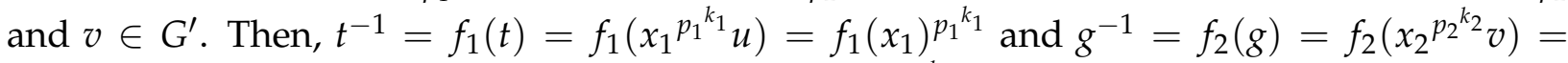
$f_{2}\left(x_{2}\right)^{p_{2} k_{2}}$. Set $y_{1}=f_{1}\left(x_{1}\right)^{-1}, y_{2}=f_{2}\left(x_{2}\right)^{-1}$. So $t=y_{1} p_{1}^{k_{1}}, y_{1} \in Z(T) \cap T_{p_{1}},\left\{y_{1}\right\} \cap T^{\prime}=1$ and 
$g=y_{2} p_{2}^{k_{2}}, y_{2} \in Z(G) \cap G_{p_{2}},\left\{y_{2}\right\} \cap G^{\prime}=1$. By [7], $y_{1} T^{\prime}$ generates a direct factor of $T_{p_{1}} / T^{\prime}$ and $y_{2} G^{\prime}$ of $G_{p_{2}} / G^{\prime}$ too, say

$$
T_{p_{1}} / T^{\prime}=\left\{y_{1} T^{\prime}\right\} \times H_{p_{1}} / T^{\prime} \text { and } G_{p_{2}} / G^{\prime}=\left\{y_{2} G^{\prime}\right\} \times H_{p_{2}} / G^{\prime} .
$$

Since $\left\{y_{1}\right\} \cap T^{\prime}=1, T=\left\{y_{1}\right\} \times\left(H_{p_{1}} T_{p_{1}^{\prime}}\right)$ is a direct decomposition of $T$, it follows that $T$ has an abelian direct factor and $G$ too, if the mapping $\left\langle f_{1}, f_{2}\right\rangle$ is not onto.

Let $C^{*}$ be the set of all central automorphisms of $(T, G, \partial)$ fixing $Z(T, G, \partial)$ element wise.

Theorem 3. If $T$ is a finite $p_{1}$-group and $G$ is a finite $p_{2}$-group, then $C^{*}=I_{n n}(T, G, \partial)$ if and only if $T, G$ are abelian or $T$ and $G$ are nilpotent of class 2 and $Z(T), Z(G)$ are cyclic.

Proof. We have

$$
C_{A u t_{C}(T, G, \partial)}(Z(T, G, \partial)) \cong \operatorname{Hom}((T, G, \partial) / Z(T, G, \partial), Z(T, G, \partial)) .
$$

Since every element of $\left\langle f_{1}, f_{2}\right\rangle \in C^{*}$ fixes each element of $Z(T, G, \partial)$, for $\left\langle f_{1}, f_{2}\right\rangle \in C^{*}$, the map $\left\langle\sigma_{1} f_{1}, \sigma_{2} f_{2}\right\rangle$ of $(T, G, \partial) / Z(T, G, \partial)$ to $Z(T, G, \partial)$ defined by $\sigma_{1 f_{1}}: T / T^{G} \rightarrow T^{G}$ such that $\sigma_{1 f_{1}}\left(t T^{G}\right)=t^{-1} f_{1}(t)$ and $\sigma_{2 f_{2}}\left(g\left(S t_{G}(T) \cap Z(G)\right)\right)=g^{-1} f_{2}(g)$ are well defined. It is obvious to see that $\sigma_{1}: f_{1} \rightarrow \sigma_{1 f_{1}}$ and $\sigma_{2}: f_{2} \rightarrow \sigma_{2 f_{2}}$ are injective homo morphisms. Now for each

$$
\left\langle h_{1}, h_{2}\right\rangle \in \operatorname{Hom}((T, G, \partial) / Z(T, G, \partial), Z(T, G, \partial)),
$$

the crossed module map $\left\langle f_{1}, f_{2}\right\rangle$ defined by $f_{1}(t)=t h_{1}\left(t T^{G}\right)$ for all $t \in T$ and $f_{2}(g)=$ $g h_{2}\left(g\left(S t_{G}(T) \cap Z(G)\right)\right)$ for all $g \in G$ is a central automorphism fixing $Z(T, G, \partial)$ element-wise and $\left\langle\sigma_{1}, \sigma_{2}\right\rangle_{\left\langle f_{1}, f_{2}\right\rangle}=\left\langle h_{1}, h_{2}\right\rangle$. It follows $\left\langle\sigma_{1}, \sigma_{2}\right\rangle$ is a crossed module isomorphism and

$$
C^{*} \cong \operatorname{Hom}((T, G, \partial) / Z(T, G, \partial), Z(T, G, \partial)) .
$$

Now, suppose first that $C^{*} \cong I_{n n}((T, G, \partial))$ and $T, G$ are non-abelian. If $t \in T$ and $g \in G$, then the inner automorphism $\left\langle\theta_{1}, \theta_{2}\right\rangle\langle t, g\rangle$ induced by $t, g$ is a central automorphism and so $[x, t]=$ $x^{-1} \theta_{1 t}(x) \in T^{G}$ for all $x \in T$ and $[y, g]=y^{-1} \theta_{2 g}(y) \in S t_{G}(T) \cap Z(G)$ for all $y \in G$. This shows that $T$ and $G$ are nilpotent of class 2. Since $T$ is nilpotent of class $2, \exp \left(T / T^{G}\right)=\exp \left(T^{\prime}\right)=p_{1}^{c_{1}}$ for some natural number $c_{1}$, and $\exp \left(G / S t_{G}(T) \cap Z(G)\right)=\exp \left(G^{\prime}\right)=p_{2}^{c_{2}}$. Let $T / T^{G}$ and $T^{G}$ have ranks $r_{1}$ and $s_{1}$, respectively, and $G / S t_{G}(T) \cap Z(G)$ and $S t_{G}(T) \cap Z(G)$ have ranks $r_{2}$ and $s_{2}$, but $T$ and $G$ are nilpotent of class 2, it follows from [5] that $T^{G}$ and $S t_{G}(T) \cap Z(G)$ are cyclic. Conversely, if $T$ and $G$ are abelian, then it is clear that $C^{*} \cong I_{n n}((T, G, \partial))=\langle 1,1\rangle$. Assume that $T$ and $G$ are nilpotent of class 2 and $Z(T)$ and $Z(G)$ are cyclic. Since $T / T^{G}$ and $G / Z(G)$ are abelian $p_{1}$ and $p_{2}$-groups of exponent $\left|T^{\prime}\right|$ and $\left|G^{\prime}\right|, T^{\prime}$ and $G^{\prime}$ are cyclic, $C^{*} \cong I_{n n}((T, G, \partial))$, since $T$ and $G$ are nilpotent of class $2, I_{n n}((T, G, \partial)) \leq C^{*}$. Hence, $C^{*} \cong I_{n n}((T, G, \partial))$.

Theorem 4. For any non-abelian groups $T$ and $G$ the restriction of the Adeny-Yen crossed module map $\left\langle\varphi_{1}, \varphi_{2}\right\rangle: C^{*} \rightarrow \operatorname{Hom}((T, G, \partial),(Z(T, G, \partial))$ is a crossed module homomorphism.

Proof. Suppose that $\left\langle\alpha_{1}, \theta_{1}\right\rangle$ and $\left\langle\alpha_{2}, \theta_{2}\right\rangle \in C^{*}$. On the other hand, we have the following diagrams
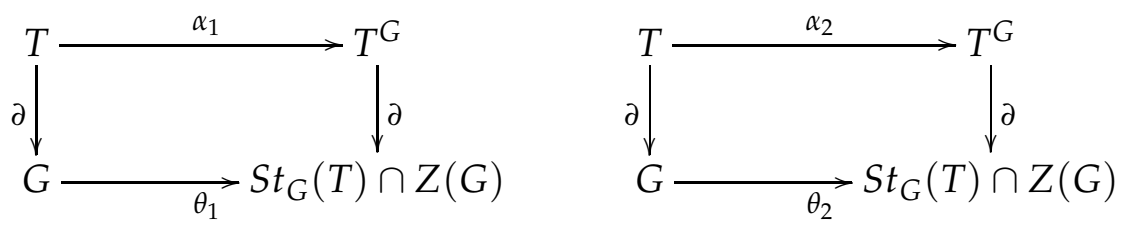
Then, for any $t \in T, g \in G$ we have $\varphi_{1\left\langle\alpha_{1}, \theta_{1}\right\rangle\left\langle\alpha_{2}, \theta_{2}\right\rangle}(t)=t^{-1}\left(\alpha_{1} \circ \alpha_{2}\right)(t)=t^{-1}\left(\alpha_{1}\left(\alpha_{2}(t)\right)\right.$ and

$$
\begin{aligned}
\varphi_{1\left\langle\alpha_{1}, \theta_{1}\right\rangle} \circ \varphi_{1\left\langle\alpha_{2}, \theta_{2}\right\rangle}(t) & =\varphi_{1\left\langle\alpha_{1}, \theta_{1}\right\rangle}\left(t^{-1} \alpha_{2}(t)\right)=\left(t^{-1} \alpha_{2}(t)\right)^{-1} \alpha_{1}\left(t^{-1} \alpha_{2}(t)\right) \\
& =\alpha_{2}\left(t^{-1}\right) t \alpha_{1}\left(t^{-1}\right) \alpha_{1}\left(\alpha_{2}(t)\right)=\alpha_{2}\left(t^{-1}\right) t^{-1} \alpha_{1}\left(\alpha_{2}(t)\right)=t^{-1} \alpha_{1}\left(\alpha_{2}(t)\right)
\end{aligned}
$$

Moreover, $\varphi_{1\left\langle\alpha_{1}, \theta_{1}\right\rangle\left\langle\alpha_{2}, \theta_{2}\right\rangle}(g)=g^{-1}\left(\alpha_{1} \circ \alpha_{2}\right)(g)=g^{-1}\left(\alpha_{1}\left(\alpha_{2}(g)\right)\right.$ and

$$
\begin{aligned}
\varphi_{1\left\langle\alpha_{1}, \theta_{1}\right\rangle} \circ \varphi_{1\left\langle\alpha_{2}, \theta_{2}\right\rangle}(g) & =\varphi_{1\left\langle\alpha_{1}, \theta_{1}\right\rangle}\left(g^{-1} \alpha_{2}(g)\right)=\left(g^{-1} \alpha_{2}(g)\right)^{-1} \alpha_{1}\left(g^{-1} \alpha_{2}(g)\right) \\
& =\alpha_{2}\left(g^{-1}\right) g \alpha_{1}\left(g^{-1}\right) \alpha_{1}\left(\alpha_{2}(g)\right)=g \alpha_{2}\left(g^{-1}\right) \alpha_{1}\left(g^{-1}\right) \alpha_{1}\left(\alpha_{2}(g)\right) \\
& =g^{-1} \alpha_{1}\left(g^{-1}\right) \alpha_{1}\left(\alpha_{2}(g)\right)=g^{-1} \alpha_{1}\left(\alpha_{2}(g)\right) .
\end{aligned}
$$

So we have $\left\langle\varphi_{1}, \varphi_{2}\right\rangle_{\left\langle\alpha_{1}, \theta_{1}\right\rangle\left\langle\alpha_{2}, \theta_{2}\right\rangle}=\left\langle\varphi_{1}, \varphi_{2}\right\rangle\left\langle\alpha_{1}, \theta_{1}\right\rangle \circ\left\langle\varphi_{1}, \varphi_{2}\right\rangle\left\langle\alpha_{2}, \theta_{2}\right\rangle$.

Theorem 5. If $T$ and $G$ are purely non-abelian group and $A u t_{C}(T, G, \partial)=C^{*}$, then

$$
\operatorname{Aut}_{C}(T, G, \partial) \cong \operatorname{Hom}((T, G, \partial), Z(T, G, \partial)) \text {. }
$$

Proof. Since $T$ and $G$ are purely non-abelian, so by Theorem 2 the Adeny-Yen crossed module map from $\operatorname{Aut}_{C}(T, G, \partial)$ to $\operatorname{Hom}((T, G, \partial), Z(T, G, \partial))$ is a bijection. But also $\operatorname{Aut}_{C}(T, G, \partial)=$ $C^{*}$, and thus by Theorem $4,\left\langle\varphi_{1}, \varphi_{2}\right\rangle$ is a crossed module homomorphism. Therefore, $\operatorname{Aut}_{C}(T, G, \partial) \cong \operatorname{Hom}((T, G, \partial), Z(T, G, \partial))$.

Theorem 6. Let $T$ and $G$ be purely non-abelian groups such that

$$
A u t_{C}(T, G, \partial)=Z\left(I_{n n}(T, G, \partial)\right)
$$

Then

$$
\operatorname{Aut}_{C}(T, G, \partial) \cong \operatorname{Hom}((T, G, \partial), Z(T, G, \partial)) \text {. }
$$

Proof. $Z\left(I_{n n}(T, G, \partial)\right)$ is a sub-crossed module of $I_{n n}(T, G, \partial)$, which fixes $Z(T, G, \partial)$ pointwise, so $A u t_{C}(T, G, \partial)=C^{*}$. Thus, by Theorem 5 we have the desired conclusion.

Theorem 7. Let $T$ and $G$ be non-abelian group such that $A u t_{C}(T, G, \partial)=Z\left(I_{n n}(T, G, \partial)\right)$. Then either $T$ and $G$ are purely non-abelian or $T$ and $G$ have purely non-abelian subgroups $T_{1}$ and $G_{1}$, with $\left|Z\left(T_{1}\right)\right|$ and $\left|Z\left(G_{1}\right)\right|$ odd such that $T=C_{2} \times T_{1}, G=C_{2} \times G_{1}$.

Proof. Suppose that on the contrary $T=A \times T_{1}$ and $G=B \times G_{1}$, where $T_{1}$ and $G_{1}$ are purely non-abelian, $A, B$ are non-trivial abelian and either $A \neq C_{2}$ or $A=C_{2}$ or $B \neq C_{2}$ or $B=C_{2}$ and $\left|Z\left(T_{1}\right)\right|$ and $\left|Z\left(G_{1}\right)\right|$ are even. In these case, we claim $(T, G, \partial)$ has a central automorphism that is not inner, on the other hand $A u t_{C}(T, G, \partial) \neq Z\left(I_{n n}(T, G, \partial)\right)$. If $A \neq C_{2}$ and $B \neq C_{2}$ and $\left(\varphi_{1}, \varphi_{2}\right) \in \operatorname{Aut}(A, B, \partial)=\operatorname{Aut}_{C}(A, B, \partial)$ is non-trivial, then for any $\left(a, t_{1}\right) \in A \times T_{1}$ and $\left(b, g_{1}\right) \in B \times G_{1}$ maps $\left(a, t_{1}\right) \rightarrow\left(\varphi_{1}(a), t_{1}\right)$ and $\left(b, g_{1}\right) \rightarrow\left(\varphi_{2}(b), g_{1}\right)$ give an automorphism of $(T, G, \partial)$ that is central but not inner. If $A=C_{2}$ and $B=C_{2}$ and $|Z(A)|$ and $|Z(B)|$ are even, take $z_{1} \in Z(A)$ and $z_{2} \in Z(B)$ with $z_{1}$ and $z_{2}$ or order 2 , so maps $\left(1, t_{1}\right) \rightarrow\left(1, t_{1}\right)$, $\left(1, g_{1}\right) \rightarrow\left(1, g_{1}\right),\left(a, t_{1}\right) \rightarrow\left(a, z_{1} t_{1}\right)$ and $\left(b, g_{1}\right) \rightarrow\left(b, z_{2} g_{2}\right)$ define an automorphism of $(T, G, \partial)$ that is central but not inner, since $(a, 1) \rightarrow\left(a, z_{1}\right)$ and $(b, 1) \rightarrow\left(b, z_{2}\right)$. 
Corollary 1. Let $p_{1}, p_{2}$ be primes and $T$ and $G$ be non-abelian $p_{1}$-group and $p_{2}$-group respectively such that $A u t_{C}(T, G, \partial)=Z\left(I_{n n}(T, G, \partial)\right)$. Then $T$ and $G$ are purely non-abelian.

Theorem 8. Let $p_{1}, p_{2}$ be primes, and $T$ and $G$ are non-abelian $p_{1}$-group and $p_{2}$-group respectively such that $\operatorname{Aut}_{C}(T, G, \partial)=Z\left(I_{n n}(T, G, \partial)\right)$. Then

$$
\operatorname{Aut}_{C}(T, G, \partial) \cong \operatorname{Hom}((T, G, \partial), Z(T, G, \partial)) .
$$

Proof. By Theorem 1, $T$ and $G$ are purely non-abelian and since

$$
\left.A u t_{C}(T, G, \partial) \cong Z\left(I_{n n}(T, G, \partial)\right)\right)
$$

is subcrossed module of $I_{n n}(T, G, \partial)$, which fixes $Z(T, G, \partial)$ point-wise, $A u t_{C}(T, G, \partial)=C^{*}$. Now, by Theorem 5 we have $A u t_{C}(T, G, \partial) \cong \operatorname{Hom}((T, G, \partial), Z(T, G, \partial))$.

Corollary 2. Let $p_{1}, p_{2}$ be primes, and $T$ and $G$ be finite $p_{1}$-group and $p_{2}$-group respectively such that $A u t_{C}(T, G, \partial)=C^{*}$. Then, $T$ and $G$ are purely non-abelian.

Theorem 9. Let $p_{1}, p_{2}$ be primes, and $T$ and $G$ be finite $p_{1}$-group and $p_{2}$-group respectively such that $\operatorname{Aut}_{C}(T, G, \partial)=C^{*}$. Then

$$
\operatorname{Aut}_{C}(T, G, \partial) \cong \operatorname{Hom}((T, G, \partial), Z(T, G, \partial)) .
$$

Proof. By Corollary 2, $T$ and $G$ are purely non-abelian. Since $A u t_{C}(T, G, \partial)=C^{*}$, then by Theorem 5 we have $A u t_{C}(T, G, \partial) \cong \operatorname{Hom}((T, G, \partial), Z(T, G, \partial))$.

\section{REFERENCES}

[1] Adney J.E., Yen T. Automorphisms of a p-group. Illinois J. Math. 1965, 9 (1), 137-143.

[2] Alp M., Wensley C.D. Automorphisms and homotopies of groupoids and crossed modules. Appl. Categ. Structures 2010, 18 (5), 473-504. doi:10.1007/s10485-008-9183-y

[3] Alp M. Actor of crossed module of algebroids. In: Proceedings of the 16th International Conference of the Jangjeon Mathematical Society, 6-15, Jangjeon Math. Soc., Hapcheon, 2005.

[4] Conduché D., Rodriguez-Fernandez C. Non-abelian tensor and exterior products module q and universal q-center relative extension. J. Pure Appl. Algebra 1992, 78 (2), 139-160.

[5] Curran M.J., Mccaughan D.J. Central automorphisms that are almost inner. Comm. Algebra 2001, 29 (5), $2081-$ 2087. doi:10.1081/AGB-100002170

[6] Doncel-Juárez J.L., R.-Grandjeán L.-Valcárcel A. q-perfect crossed modules. J. Pure Appl. Algebra 1992, 81 (3), 279-292.

[7] Kaplansky I. Infinite abelian groups. University of Michigan Press, Ann Arbor, 1954.

[8] Norrie K. Actions and automorphisms of crossed modules. Bull. Soc. Math. France 1990, 118 (2), $129-146$. doi:10.24033/bsmf.2140

[9] Whitehead J.H.C. Combinatorial homotopy II. Bull. Amer. Math. Soc. 1949, 55, 453-496. doi:10.1090/S0002-99041949-09213-3 
Аехгані М.А., Аавваз Б. Про иентральні атоморфізми перехресних модулів // Карпатські матем. публ. — 2018. - Т.10, №2. - С. 288-295.

Перехресний модуль $(T, G, \partial)$ складається з групового гомоморфізму $\partial: T \rightarrow G$ з дією $(g, t) \rightarrow g_{t}$ з $G$ на $T$, яка задовольняє $\partial(g t)=g \partial(t) g^{-1} \mathrm{i}^{\partial(s)} t=s t s^{-1}$ для всіх $g \in G$ i $s, t \in T$. Термін перехресного модуля введено $\Delta ж$. Х. К. Уайтхедом у його роботі з комбінаторики теорії гомотопій. Перехресні модулі і ї застосування відіграють дуже важливу роль в теорії категорій, теорії гомотопій, гомології і когомологиї груп, алгебрі, К-теорії тощо. У даній роботі визначено відображення Адені-Єна перехресних модулів і центральні автоморфізми перехресних модулів. Якшо $C^{*}-$ множина всіх центральних автоморфізмів перехресних модулів $(T, G, \partial)$, які поточково фіксують $Z(T, G, \partial)$, то отримано необхідну і достатню умови щоб $C^{*}=I_{n n}(T, G, \partial)$. У цьому випадку доведено $A u t_{C}(T, G, \partial) \cong \operatorname{Hom}((T, G, \partial), Z(T, G, \partial))$. Крім того, якщо $\left.A_{u t}(T, G, \partial) \cong Z\left(I_{n n}(T, G, \partial)\right)\right)$, то отримані також певні результати в цьому напрямку.

Ключові слова і фрази: перехресний модуль, центральний автоморфізм. 